\title{
AN EXAMPLE OF A FIXED POINT FREE HOMEOMORPHISM OF THE PLANE WITH BOUNDED ORBITS
}

\author{
BY STEPHANIE M. BOYLES 1
}

In 1912 L. E. J. Brouwer proved his famous translation theorem [3] which states that if $h$ is an orientation preserving homeomorphism of $E^{2}$ onto itself having no fixed points, then $h$ is a translation. By a translation, Brower meant that for each $x$ in $E^{2}, h^{n}(x) \rightarrow \infty$ as $n \rightarrow \pm \infty$; that is, the orbit of every point is unbounded. The question arose as to whether or not any homeomorphism of $E^{2}$ onto itself with the property that the orbits of every point is bounded must have a fixed point. This eventually became known as the bounded orbit problem [2].

In this short note we wish to announce the existence of an orientation reversing fixed point free homeomorphism $h$ of $E^{2}$ onto itself having the property that the orbit of every point is bounded [1]. We note that the orbit of a point $p$ is the set of all iterates $h^{n}(p)$, where $n$ is an integer. The homeomorphism we construct can be briefly described as follows. On the complement of the strip $|x|<1, h$ is a reflection across the $y$-axis. Between the lines $x=-1$ and $x=1$ we first define $h$ on positive images of the $\operatorname{arc} A=\{(x, y):|x| \leqslant 1$ and $y=0\}$. For all integers $m \geqslant 0$ and $k \geqslant 1$, let

$$
v_{ \pm m, 0}=( \pm m /(m+1), 0)
$$

and

$$
v_{ \pm m, k}=\left( \pm m /(m+1), \sum_{i=1}^{k} 1 /(m+i)\right)
$$

For all integers $j$ and nonnegative integers $k$, define

$$
h\left(v_{j, k}\right)=v_{(-1)^{k+1}-j, k+1}
$$

Extend $h$ linearly on each line segment $\left[v_{j-1, k}, v_{j, k}\right]$ by defining

$$
h\left(\left[v_{j-1, k}, v_{j, k}\right]\right)=\left[h\left(v_{j-1, k}\right), h\left(v_{j, k}\right)\right],
$$

Received by the editors May 5, 1980.

1980 Mathematics Subject Classification. Primary 54H25, 55M20.

Key words and phrases. Fixed point free homeomorphism, bounded orbits, orientation preserving, orientation reversing.

${ }^{1}$ This is part of the author's doctoral dissertation at the University of Florida under the supervision of Professor Gerhard X. Ritter. 
for all integers $j$ and nonnegative integers $k$ (Figure 1). Observe that for nonnegative $j$,

$$
h^{k}\left(v_{j, 0}\right)=v_{(-1)^{k}(j+k), k}=\left((-1)^{k}(j+k) /(j+k+1), \sum_{i=1}^{k} 1 /(j+k+i)\right) .
$$

Since $\Sigma_{i=1}^{k} 1 /(j+k+i) \rightarrow \log 2$ as $k \rightarrow+\infty$, the images of points of form $v_{|j|, 0}$ are bounded. When $j$ is negative,

$$
h^{k-j}\left(v_{j, 0}\right)=v_{(-1)^{k} k, k-j}
$$

forcing all points of form $v_{j, 0}$ to have bounded orbits. Hence, with this construction every point on $A$ has a bounded orbit even though the orbit of $A$ itself is unbounded.

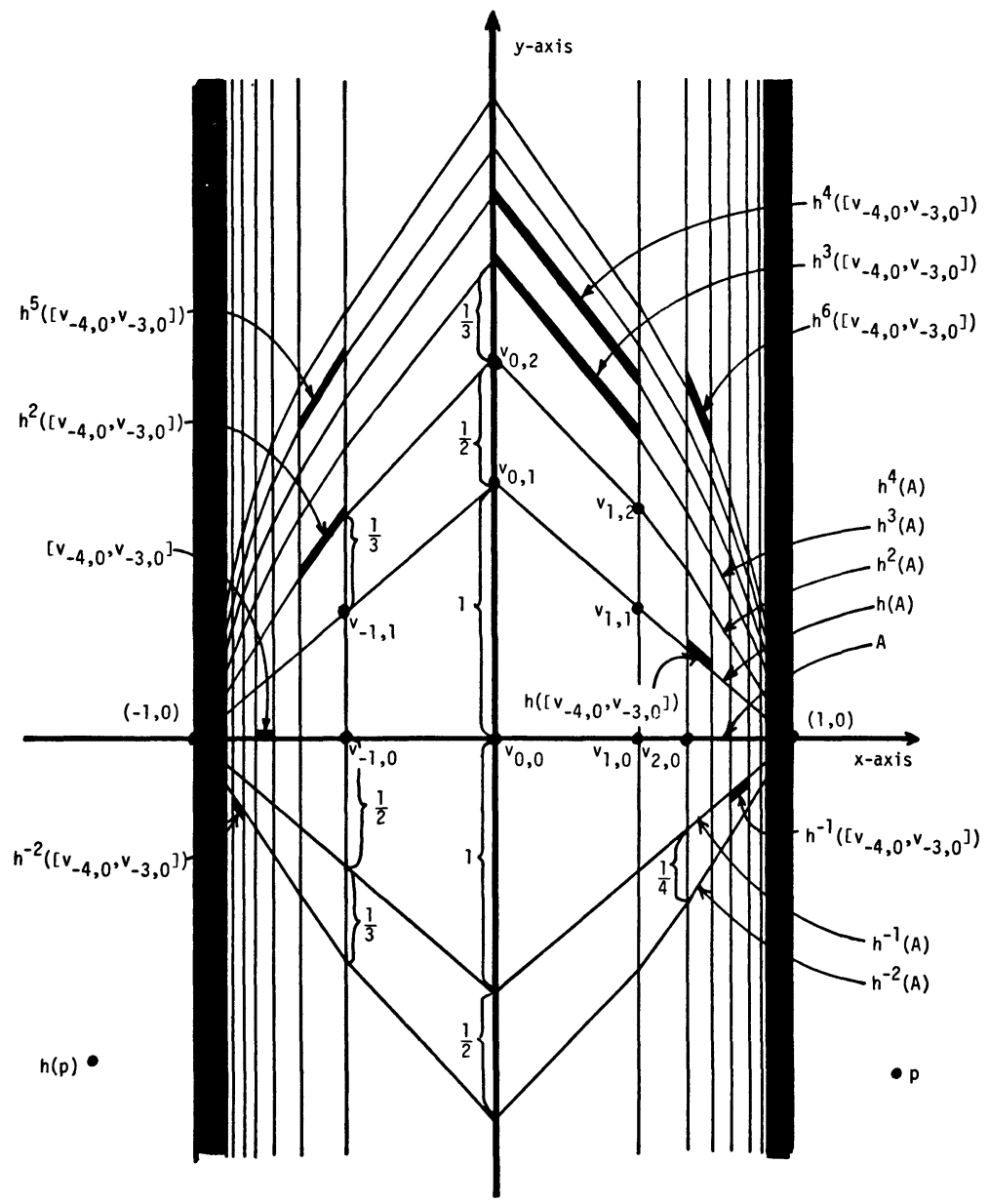

Figure 1 
To define the homeomorphism between $h^{k-1}(A)$ and $h^{k}(A)$ so that points on these regions have bounded orbits, we use the fact that the positive orbits of points on $h^{k}(A)$ are bounded. The homeomorphism is constructed so that under successive applications of $h$, points between $h^{k-1}(A)$ and $h^{k}(A)$ are pushed away from the images of $h^{k-1}(A)$ and toward the images of $h^{k}(A)$. For every point $p$, there is an integer $n$ (depending on $p$ ) such that the distance from $h^{n}(p)$ to $h^{n}\left(h^{k-1}(A)\right)$ exceeds the distance from $h^{n}(p)$ to $h^{n}\left(h^{k}(A)\right)$. Letting $q$ denote the point on $h^{n}\left(h^{k}(A)\right)$ directly above $h^{n}(p), h$ is defined so that $h^{m}\left(h^{n}(p)\right)$ lies directly beneath $h^{m}(q)$ for every nonnegative integer $m$. Thus, since the positive orbit of $q$ is bounded, the positive orbit of $h^{n}(p)$ must also be bounded.

For a point $p$ below the $x$-axis, we define $h(p)=-h^{-1}(-p)$. Hence, points below the $x$-axis are controlled under applications of $h^{-1}$ in the same way that points above the $x$-axis are controlled under applications of $h$.

An open question relating to this problem can be stated as follows. Let $h$ be an orientation reversing homeomorphism of $E^{2}$ onto itself having the property that the orbit of every point is bounded. Define $h$ to be bounded at $p$ if there exists an open set $U$ containing the point $p$ such that the orbit of $U$ is bounded. Let $D$ be the set of all points $p$ such that $h$ is bounded at $p$. It can be shown that $D$ is dense and open in $E^{2}$. In the example we give, $D$ has infinitely many components. The question as to whether $D$ can have finitely many components without $h$ having a fixed point remains unsolved. ${ }^{2}$ Even when $D$ has just two components the answer to this question is not known.

\section{BIBLIOGRAPHY}

1. S. Boyles, A counterexample to the bounded orbit conjecture, Trans. Amer. Math. Soc. (to appear).

2. B. L. Brechner and R. D. Mauldin, Homeomorphisms of the plane, Pacific J. Math. 59 (1975), 375-381. MR 52 \#9199. 39-54.

3. L. E. J. Brouwer, Beweis des ebenen Translationesatzes, Math. Ann. 72 (1912),

DEPARTMENT OF MATHEMATICS, UNIVERSITY OF FLORIDA, GAINESVILLE, FLORIDA 32611

${ }^{2}$ This problem was communicated to me by Professor R. Dan Mauldin. 